\title{
Lessons learned from five years of student self-directed experimental projects in the advanced lab
}

\author{
Melissa Eblen-Zayas ${ }^{1} \&$ Ryan C Terrien ${ }^{1}$ \\ ${ }^{I}$ Department of Physics \& Astronomy, Carleton College, One North College St., Northfield, MN 55057, USA
}

\begin{abstract}
At Carleton College, a self-directed group project is the capstone of the "Contemporary Experimental Physics" course. Instructors in this course do not prescribe topics or experimental implementation, and a wide array of projects results. We report here on trends and lessons learned after five classes with this project (44 experiments). We compile project outcomes as measured by evaluations and student and instructor reflections. We consider how these outcomes are affected by topic source (instructor-suggested or independent), sub-field of physics, and the relative emphasis on hardware design or analytical complexity. Combined with individual case studies and topic examples, these data will serve as a valuable reference for future implementation of open-ended experimental projects in similar courses.
\end{abstract}

\section{INTRODUCTION}

With growing recognition that laboratories are more effective when their goal is to teach experimental skills rather than reinforcing physics concepts taught during class [1], providing students the opportunity to engage in the process of designing and executing their own experiments is increasingly important. Giving students practice with a range of experimental skills, including design, modeling, and technical skills, is consistent with the American Association of Physics Teaching recommendations for the undergraduate laboratory curriculum [2]. At the introductory level, curricula such as the Investigative Science Learning Environment [3] emphasize experimental design. In the advanced lab, multi-week projects can give students an authentic introduction to the practices routinely used in physics research settings [4]. Laboratory project work ranges from projects where faculty provide a menu of possible problems to be explored (e.g. the course described in [5]) to projects where students choose the topic for themselves, as is the case in our advanced lab course.

We invite students to choose their own topics for the multi-week final projects because we want students to have the opportunity to design their own experiment, including choice of what question they want to explore. Even students who engage in undergraduate research experiences often do not have much opportunity to engage in research project goal setting and they are not asked to develop contingency plans if things do not go as expected [6]. These activities, however, are key aspects of experimental research, including experimental design; therefore we wanted students to experience these activities in the curriculum. Our objective is that through the project portion of the class students will gain experience with the process of designing and carrying out an experiment. The focus is not on obtaining conclusive results, and some projects never reach the point where students are able to collect data to explore the questions they proposed. Although results are not the goal, some projects do seem to give students a more satisfying sense of progress or contribute to a better understanding of the experimental design process. Projects that effectively foster student understanding and appreciation of the process of designing and carrying out an experiment are deemed successful projects in this course.

Reviewing the 44 different final projects that 112 students have completed (in groups) over the past five years in the advanced lab course at our institution, we reflect on the topic choices students make and outcomes of the projects, and provide case studies of several successful projects. In addition, we describe how the scaffolding of the projects has evolved.

\section{Context}

\section{A. Course}

Contemporary Experimental Physics is a ten-week advanced laboratory class, typically taken by physics majors in the junior year. Prior to taking this course, students have taken at least two intermediate-level lab courses. The advanced lab course includes class meetings three times per week and a weekly four-hour lab. Students are expected to work on their experimental projects outside of the designated lab time as well. In the first part of the course, students complete three two-week labs, designed by the instructor, which are significantly less scripted than what students have seen in intermediate-level lab courses. In the second part of the course, students design and carry out the experimental project of their choosing.

The four stated learning goals for the course are: 1) That students will be able to estimate the quality of the measured data and compare measured data with predictions from a model or from previous work; 2) That students will be able to collaborate with peers to build or assemble a measurement system from a given set of components, test and revise the measurement system if it does not work as 
expected, and keep a clear record of their work as they do this; 3) That students will be able to clearly and convincingly communicate experimental work and results to an appropriate audience, either orally or in writing; 4) That students will demonstrate the ability to be reflective on the practice of experimental physics. Particular skills or experiments are not a focus of this course. Rather, the aim is for students to gain an appreciation for the process of doing experimental work. The final project is particularly focused on helping the students meet learning goals 2) and 4).

\section{B. Project}

The final project is designed to give students an opportunity to develop their experimental design skills. Students may propose projects on any topic that meets the following guidelines: 1) the project can be carried out primarily with existing equipment and resources in the department (although a budget is available for small purchases); 2) the project must include an experimental challenge that is not trivial (so analyzing existing data sets is not appropriate for this project); and 3) the project is designed to test or build on results in the published literature. The third guideline was added after several groups proposed projects that focused on building apparatus, and the students relied on do-it-yourself websites rather than peer-reviewed literature as the basis for their work; these projects were largely unsuccessful in helping students gain an understanding of the experimental design process.

The projects are carried out in groups of two or three students. Instructors assign students to groups, and students work with the same group for the entire course. Students do have the option of letting the instructor know if there are students with whom they do not work well based on past experience, but they do not select their own groups. Working with the same group for the entire course means that group members identify difficult dynamics long before the project work begins in lab, and course activities are designed to explicitly foster discussion of group dynamics and help students address difficulties.

The final project is introduced in the first week of the course. Students are given a list of 12-16 topics with a reference to an article that can serve as a starting point for exploring the literature, but students are not constrained to choosing from this list. Over the last five years, fewer than half of the groups have selected a topic from the instructor's list. Groups develop their final project proposal over the first five weeks of the course, including library instruction, database searching activities, and practice with literature review. After the first draft of the proposal (due week four), students revise their proposal based on peer review and submit a final proposal in week five. Following reflection on the proposal process and upcoming challenges, students conference with the instructor during the 6th week of the course, and work on the projects during class time and during laboratory in the last four weeks of the course. The final project deliverables include: a preliminary and a revised final project proposal, a poster (presented as a group), an individual journal-style write-up, and an electronic lab notebook that is kept by the group [7].

\section{Project Topics}

Students are often both daunted and excited by the prospect of being able to choose their own topic. Reviewing the past five years, final projects have most often explored four areas of physics: fluids, waves, thermal physics, and materials physics (both hard and soft matter). In our department, all of these areas are elective courses without a lab. In short, many students seem to use the projects to explore areas that are not at the core of our curriculum.

Although topics vary widely, a common challenge for students is to define the scope of their projects during the proposal stage. This project is the first time many students approach the literature knowing they will have to rely on it in their project work. Increasing the scaffolding of the proposal (more classroom discussion and instruction relevant to the proposal development and more detailed rubrics for the peer review process) has been important for supporting the development of appropriate project proposals. In recent years, student reflections indicate that defining project goals and scope is a high-level skill that they develop in the advanced lab. It is also a skill that is not developed in undergraduate research experiences [6].

\section{A. Modeling}

Although there is no "typical project", one observation is that projects on waves lend themselves to modeling more than other projects. Four of the six projects on waves included significant work with analytical/computational models. Outside of the waves projects, only two other projects had a significant modeling component (one project on diffusion and one project on conservation of angular momentum). Waves projects that included modeling were:

- Non-normal damping of banjo strings [8], [9].

- Speed of sound in liquid [10].

- Acoustic analog of QM band structure [11], [12].

- Vibrating guitar strings [13], [14].

\section{B. Complex Instrumentation}

Another notable correlation is that students engaging in projects where the first step is the construction of complex instrumentation often find that this process dominates what they can achieve in the four weeks allotted. The resulting focus on the assembly of the apparatus precludes deep or 
iterative work on the experiment itself, and leaves students with a limited experience of the experimental process. Instrument-focused projects also appear to steer students toward narrower engagement with the literature, as the class of problems they encounter are better-suited to, e.g., do-it-yourself websites.

Seven groups over the past five years have proposed building complex instrumentation as the first step of their project. Some groups have built and successfully calibrated the instrument, but none got data beyond calibration. These instrument-building projects included: a chaotic pendulum [15]; a Watt balance [16]; a bomb calorimeter; a rheometer; a spectrometer for Raman spectroscopy; a scanning tunneling microscope; and a spectrometer for Förster resonance energy transfer.

\section{Examples of Successful Projects}

As examples of successful projects compatible with the final project timeline, we highlight here the work of three groups. All of these projects fostered meaningful engagement with the experimental design process. Students gained experience in the machine shop building apparatus, collected data in a timely manner that allowed some iteration (either of apparatus design or of experimental methods), and used the published literature to put their results in context. None of these projects were based on an American Journal of Physics $(A J P)$ article that provides a detailed advanced lab activity; that seems to have contributed to the students having to think more deeply about their experimental design.

\section{A. The Leidenfrost Effect}

This project exploring the Leidenfrost effect was based on a research article [17], so an experimental approach is presented, but the implementation is not described in a pedagogical manner.

This experiment provided many variables to explore ("ratchet" topology, ramp angle, temperature, liquid, etc), and the students could control many of those variables without significant technical difficulty. The machine shop work was time-consuming, but experimental data collection was quick so there were opportunities to iterate depending on results. In this particular implementation, students turned an experimental setback (chemical contamination of the "ratchet" material) into an opportunity to test the effects of different types of contamination. In post-project reflective statements, group members discussed the importance of flexibility, and the opportunity for unexpected exploration appears to have been important for their sense of agency and ownership in this project.

\section{B. The Speed of Sound in Liquids}

This project explored the speed of sound in a liquid by measurement of optical diffraction pattern and was based on a two-page AJP Apparatus and Demonstration Note [10]. This foundational article is brief so students get a sense of possibilities for extension, but the article does not outline details.

For this experiment, neither the set-up nor the data collection were overly complicated. This meant that within the project work period students had time to iterate both the design of the apparatus and design of the experimental methods. This project also provided good opportunities to compare results to theoretical expectations. In post-project reflective statements, all group members emphasized the role of troubleshooting and iteration in their learning process.

\section{2D Fluid Flow}

The project used vertically falling soap films as a vehicle for exploring 2D fluid flow. The foundational article for this project [18] describes an apparatus that is much more complicated than what is feasible in the advanced lab, and students had to isolate essential elements of this experiment to design a suitable project.

Although the basic apparatus to produce 2D fluid flow was fairly straightforward to construct, developing a feasible method to image the fluid flow within curricular resource constraints required iteration. This project provided students a rich set of experiences with regards to data visualization. Results were both qualitative (e.g. flow lines) and quantitative (e.g. vortex shedding rate). Additionally, the experimental design was flexible enough to allow students to change several variables that impact flow. In their post-project reflections, students noted because they had to simplify experimental approaches described the in literature, they had to be creative, and the methods developed were truly their own.

\section{Metacognitive Aspects}

The advanced lab exposes students to a degree of open-ended experimental work that they have not seen in previous lab courses. To support students as they move from structured to open-ended problem solving in the laboratory, we have introduced an increasing number of metacognitive support activities over the past five years. ${ }^{1}$ When we first introduced the final projects into the course, we included no metacognitive activities. Over time, we have implemented a number of forward-looking and

\footnotetext{
${ }^{1}$ More detailed discussion of these activities can be found in [19].
} 
reflective metacognitive exercises. These encourage students to practice the regulation of their cognition and to engage in a social environment that normalizes the frustrations of open-ended experimental work [19]. These activities include written prompts that ask students to reflect on the proposal process, to predict what they forsee as the biggest challenges in their project work, and to regularly engage in goal-setting, reflection on successes and problems encountered, as well as a final reflection on the process as a whole. In addition, in-class discussion of experimental progress is also used to develop a supportive social environment for metacognition. We have found these to be invaluable tools, and have thus included them explicitly in the course goals.

\section{Conclusions and Lessons Learned}

In implementing an open-ended project in the advanced lab over five years, we have found a number of elements essential for student success. The proposal process is indispensable, allowing students to engage with the broader context of their project, to begin to develop a sense of ownership, and to gain experience working with the group with whom they will complete the project.

As part of both the proposal process and the implementation of the experiment, metacognitive activities help students identify and implement effective strategies for learning, problem-solving, and collaboration.

We consider most successful those projects that demonstrate growth in understanding and appreciation of the process of designing and carrying out an experiment, particularly those that demonstrate iterative refinement of methods and apparatus. Although this is emphasized throughout the course, we suggest emphasizing this definition of success to the students clearly and early-on.

We note, and highlight in Section IV, two essential aspects of successful projects. One is the definition and constraint of project scope, which largely takes place during the project proposal stage and which is rightly seen by many students as one of the chief "take-away" lessons from this course. Another is the availability of sufficient experimental flexibility (or contingency) to explore interesting experimental pathways even if issues arise.

While we do not observe a clear link between project outcomes and subject or topic source (i.e. instructor list or student-generated), we do observe two notable classes of projects: those relying on complicated apparatus that often get bogged down in instrument assembly/calibration, and those that afford students the opportunity to compare a rich parameter space to model predictions.

As we refine our implementation of the advanced lab, it is our hope that the observations presented here will provide useful comparisons for other implementations of final projects in the physics lab curriculum.

\section{ACKNOWLEDGEMENTS}

The authors wish to thank the other instructors who have taught lab sections of this course over the past five years: Julie Coats, Eric Hazlett, and Barry Costanzi. The authors also thank the referees for their insightful suggestions, which have helped improve this manuscript.
[1] N. G. Holmes and C. E. Wieman, Physics Today 71 (1), 38 (2018).

[2] Kozminski et al. AAPT Committee on Laboratories, AAPT Recommendations for the Undergraduate Physics Laboratory Curriculum (2014).

[3] E. Etkina, S. Murthy, and X. Zou, Am. J. Phys. 74, 979 (2006).

[4] P.W. Irving and E. C. Sayre, Phys. Rev. Special Topics-Phys. Ed. Res. 10, 010109 (2014).

[5] D. R. Dounas-Frazer, J. T. Stanley, and H. J. Lewandowski, Phys. Rev. Phys. Ed. Res. 13, 020136 (2017).

[6] N. G. Holmes and C. E. Wieman, Phys. Rev. Phys. Ed. Res. 12, 020103 (2016).

[7] M. Eblen-Zayas, 2015 Conference on Laboratory Instruction Beyond the First Year of College Proceedings, 28, (2015).

[8] D. Politzer, Am. J. Phys. 83, 395 (2015)
[9] L. A. Stephey and T. R. Moore, J. of the Acoustical Society of America 124, 3276 (2008).

[10] D. A. Luna, M. A. Real and D. V. Duran, Am. J. Phys. 70, 874 (2002).

[11] D’Onofrio et. al, Am. J. Phys. 84, 841 (2016)

[12] S. A. Hilbert and H. Batelaan, Am. J. Phys. 75, 1003 (2007).

[13] P. Perov, W. Johnson, and N. Perova-Mello, Am. J. Phys. 84, 38 (2016)

[14] S. B. Whitfield and K. B. Flesch, Am. J. Phys. 82, 102 (2014).

[15] R. DeSerio, Am. J. Phys. 71, 250 (2003)

[16]L.S. Chao et al., Am. J. Phys. 83, 913 (2015)

[17] A. Grounds, R. Still, and K. Takashina, Scientific reports 2, 720 (2012).

[18]M.A. Rutgers, X.L. Wu, W.B. Daniels, Review of Scientific Instruments 72, 3025 (2001).

[19] M. Eblen-Zayas, 2016 Physics Education Research Conference Proceedings, 104, (2016). 